\title{
Níveis tensionais de adultos indígenas Suruí, Rondônia, Brasil
}

\author{
Blood pressure levels of Suruí indigenous adults \\ in Rondônia, Brazil
}

Felipe Guimarães Tavares ${ }^{1}$

Carlos Everaldo Alvares Coimbra Junior ${ }^{1}$

Andrey Moreira Cardoso ${ }^{1}$

${ }^{1}$ Escola Nacional de Saúde Pública, Fundação Oswaldo Cruz. R. Leopoldo Bulhões 1480, Manguinhos. 21041-210 Rio de Janeiro RJ. felipetavares@ensp.fiocruz.br

\begin{abstract}
Indigenous peoples in Brazil are experiencing rapid epidemiologic and nutritional transition, with non-communicable diseases such as hypertension emerging in their health profile. A cross-sectional study was conducted in 9 Surui Indian villages ( $n-251$ subjects) in Rondônia, Brazilian Amazon, in 2005, in order to assess blood pressure levels in adults ( $>20$ years of age), as well as to investigate its possible relationship with nutritional and socioeconomic status (SES). Mean systolic blood pressure (SBP) and diastolic blood pressure (DBP) were higher in men. On average, an increase in SBP of $7.9 \mathrm{mmHg}$ and of $1.4 \mathrm{mmHg}$ in women and men, respectively, was detected. SBP was positively correlated with waist-hip ratio (WHR) in both sexes and with age in women. DBP showed statistically significant correlations with all anthropometric variables, except height and arm muscle area. The prevalence of hypertension was $2.8 \%$ (M: 2.4\%, F: 3.1\%), being higher in subjects $>40$ years with PC or high WHR, especially in women and also in the group that manifested lower SES. The study concludes that hypertension is an emerging health problem among the Suruí.
\end{abstract}

Key words Blood pressure, Anthropometrics, South American Indians, Amazon, Suruí
Resumo Os povos indígenas no Brasil vivenciam acelerado processo de transição nutricional e epidemiológica, verificando-se a emergência de doenças e agravos não transmissíveis, como hipertensão arterial (HA). Realizou-se, em 2005, um estudo transversal para descrever os níveis tensionais em adultos (> 20 anos) indígenas Suruí, Rondônia, e investigar sua relação com o estado nutricional e o nível socioeconômico (SSE). Foram visitadas 9 aldeias e avaliados 251 indivíduos (87,4\% dos elegíveis). As médias de pressão arterial sistólica (PAS) e pressão arterial diastólica (PAD) foram maiores no sexo masculino e superiores às verificadas em 1988, com incremento na média da PAS de 7,9 $\mathrm{mmHg}$ e de 1,4 $\mathrm{mmHg}$, em mulheres e homens, respectivamente. A PAS correlacionou-se positivamente com a razão cintura quadril (RCQ) em ambos os sexos, e com a idade, no sexo feminino. A PAD apresentou correlações estatisticamente significativas com todas as variáveis antropométricas, exceto com estatura é área muscular do braço. A prevalência de HA foi de 2,8\% (M: 2,4\%; F: 3,1\%). Essa prevalência foi maior nos indivíduos $>40$ anos, com perímetro da cintura (PC) ou RCQ elevados, sobretudo no grupo feminino e também no grupo de mais baixo de SSE. A HA é um problema de saúde emergente entre os Suruí, devendo receber atenção do sistema de saúde e dos pesquisadores.

Palavras-chave Pressão arterial, Antropometria, Índios Sul-Americanos, Amazônia, Suruí 


\section{Introdução}

Os povos indígenas no Brasil vêm experimentando um acelerado processo de transição em saúde caracterizado pela emergência de doenças crônicas não transmissíveis, com destaque para a obesidade, diabetes mellitus e hipertensão arterial ${ }^{1-5}$. Em sua maioria, pesquisas realizadas antes da década de 1990 sobre níveis tensionais das populações indígenas residentes em aldeias reportavam valores de pressão arterial médios inferiores aos observados na população nacional não indígena e ausência de associação positiva com a idade, bem como inexistência de casos de hipertensão arterial $^{6}$. Estudos mais recentes, no entanto, têm reportado não somente a elevação dos níveis tensionais com a idade, como também a ocorrência de casos de hipertensão arterial em povos indígenas em diversas regiões do país ${ }^{2,7,8}$.

Nas últimas décadas, a Amazônia tem sido cenário de rápida expansão das últimas fronteiras demográficas e econômicas do Brasil. Este processo tem tido ampla repercussão sobre a saúde e a nutrição das populações indígenas da região. Entre os indígenas Suruí, ainda que as doenças infecciosas e parasitárias, a desnutrição proteico-calórica em crianças e a anemia ${ }^{9-12}$ tenham grande expressão no cenário epidemiológico da população, registra-se a rápida emergência da obesidade como um problema de saúde entre os adultos ${ }^{13,14}$. Esse contexto epidemiológico tem sido atribuído à crescente interação da população com a sociedade envolvente e sua maior dependência do mercado regional para sua subsistência, com profundas implicações sobre as práticas alimentares ${ }^{14}$.

Tendo em vista as transformações no cenário socioeconômico e a existência de estudo que reportou dados de pressão arterial para a população adulta Suruí ao final da década de $1980^{15}$, este trabalho tem por objetivo descrever os níveis tensionais atuais na população Suruí adulta e investigar sua relação com o estado nutricional e o nível socioeconômico atual, bem como comparar os níveis tensionais médios verificados nessa população no presente com aqueles reportados ao final da década de $1980^{15}$.

\section{População e Métodos}

Os dados aqui apresentados foram coletados no âmbito de uma pesquisa de maior abrangência que teve por objetivo investigar a epidemiologia da tuberculose, parasitoses intestinais e estado nutricional do povo indígena Suruí10,11,14,16. Na ocasião, foram também coletados dados de pressão arterial em adultos de ambos os sexos com idade $>20$ anos, que compuseram o banco de dados para a presente análise.

A pesquisa de campo na qual os dados foram coletados foi realizada entre fevereiro e março de 2005. À época, os Suruí somavam 993 indivíduos (sendo $355>20$ anos de idade) e viviam em onze aldeias situadas na Terra Indígena Sete de Setembro, localizada na divisa dos estados de Rondônia e Mato Grosso.

A pressão arterial foi aferida por um mesmo observador, utilizando-se um esfigmomanômetro de coluna de mercúrio modelo FC-110, fabricado pela Focal Corporation (Kashiwa, Japão). As aferições foram realizadas no braço direito, com manguito padrão para adulto. Durante o procedimento, os indivíduos permaneciam sentados com os pés apoiados no chão. Foram realizadas duas medidas de pressão arterial consecutivas em cada indivíduo, com intervalo aproximado de dez minutos entre elas. A pressão arterial sistólica (PAS) foi assinalada na $1^{\text {a }}$ fase de Korotkoff e a diastólica (PAD), na $5^{\mathrm{a}}$ fase. Para os fins de análise, foram considerados como valores de PAS e PAD as médias das duas medidas consecutivas, corrigidas para o perímetro do braço do indivíduo ${ }^{17}$.

Os níveis tensionais foram classificados em categorias de pressão arterial segundo os critérios recomendados pelo The Seventh Report of the Joint National Committeeon Prevention, Detection, Evaluation, and Treatment of High Blood Pressure (JNC 7) ${ }^{18}$, sendo considerado hipertenso o indivíduo que apresentasse pressão arterial sistólica $(\mathrm{PAS})>140 \mathrm{mmHg}$ e/ou pressão arterial diastólica $(\mathrm{PAD})>90 \mathrm{mmHg}$. Além disso, os indivíduos foram também categorizados nos seguintes estágios de gravidade: pressão arterial (PA) normal (PAS $<120$ mmHg e PAD $<80$ mmHg); pré-hipertensão (PAS 120-139 mmHg e PAD 80-89 mmHg); hipertensão I (PAS 140-159 mmHg e PAD 90-99 mmHg) e hipertensão II (PAS $>160 \mathrm{mmHg}$ ou PAD > $100 \mathrm{mmHg})^{18}$.

Os dados de peso, estatura, perímetro da cintura (PC), perímetro do quadril, perímetro braquial e prega cutânea triciptal foram obtidos segundo metodologia recomendada por Lohman et al. ${ }^{19}$. A partir dessas variáveis, foram calculados os seguintes indicadores nutricionais: índice de massa corporal (IMC), calculado pela fórmula peso $(\mathrm{kg}) /$ altura $\left(\mathrm{m}^{2}\right)$; a razão cintura/quadril (RCQ); as áreas muscular e de gordura do braço, estimadas, respectivamente, a partir do 
perímetro braquial e da prega cutânea triciptal e transformadas em escores z, segundo Frisancho $^{20}$. Maior detalhamento acerca da realização das medições antropométricas no campo encontra-se em Lourenço et al. ${ }^{14}$.

Para a classificação do estado nutricional em categorias de IMC foram considerados os seguintes pontos de corte: IMC $<18,5 \mathrm{~kg} / \mathrm{m}^{2}=$ baixo peso; IMC de 18,5 a $24,9 \mathrm{~kg} / \mathrm{m}^{2}=$ peso adequado; IMC de 25,0 a $29,9 \mathrm{~kg} / \mathrm{m}^{2}=$ sobrepeso e IMC $>30$ $\mathrm{kg} / \mathrm{m}^{2}=$ obesidade $^{21}$. Foram considerados pontos de corte para risco cardiovascular os valores do perímetro da cintura $(\mathrm{PC})>94 \mathrm{~cm}$ para o sexo masculino e de $>80 \mathrm{~cm}$, para o feminino; e de $\mathrm{RCQ}>1$ e $>0,85$, para os sexos masculino e feminino, respectivamente ${ }^{22}$.

A população de estudo foi categorizada do ponto de vista socioeconômico a partir do Status Socioeconômico (SSE) proposto por Lourenço et al. ${ }^{14}$, em três níveis: inferior, intermediário e superior. Esses níveis foram definidos por combinações de scores de "graus de ocidentalização" dos domicílios, com base em quatro dimensões: (1) materiais utilizados na construção da casa, incluindo piso, parede e cobertura; (2) número de dormitórios; (3) presença de utensílios domésticos (fogão, geladeira, freezer, aparelho de televisão, máquina de lavar roupas e antena parabólica) e (4) presença de mobiliário doméstico (cama de casal, sofá, guarda-roupa e mesa de jantar). Cada chefe de família foi classificado com relação ao nível socioeconômico, que foi posteriormente atribuído aos demais residentes no domicílio ${ }^{14}$.

Foram descritas as distribuições de frequências da população estudada por faixa etária, indicadores nutricionais e SSE, segundo sexo. Em seguida, foram descritas médias e respectivos intervalos de confiança da PAS e PAD por estratos das variáveis de estudo em ambos os sexos. Para esta análise, segundo categorias de IMC, foi excluída a categoria de baixo peso, na qual foram classificados apenas dois indivíduos masculinos. As diferenças de médias entre os sexos foram testadas por meio do teste $t$ de Student. As proporções foram comparadas por meio do teste quiquadrado $\left(\chi^{2}\right)$.

O grau de correlação entre as variáveis contínuas foi avaliado por meio do coeficiente de correlação de Pearson, em análises bivariadas para os sexos separadamente, testando-se a significância estatística através do teste t bi-caudal. As análises foram realizadas utilizando-se o software SPSS for Windows (versão 17.0). Considerouse o nível de significância de $5 \%$ em todos os testes realizados.
Os valores médios e as prevalências atuais de PAS e PAD foram comparados aos descritos na mesma população há cerca de dezessete anos ${ }^{15}$.

Este estudo foi aprovado pelo Comitê de Ética em Pesquisa da Escola Nacional de Saúde Pública e pela Comissão Nacional de Ética em Pesquisa (CONEP) do Conselho Nacional de Saúde. Recebeu também autorização para ingresso em terra indígena emitida pela Fundação Nacional do Índio (Funai). Antes do início da pesquisa de campo, o projeto foi apresentado às lideranças de cada aldeia para a obtenção de anuência. Além disso, obteve-se a autorização verbal do chefe de cada domicílio visitado.

\section{Resultados}

Foram visitadas 9 das 11 aldeias existentes, devido a dificuldades logísticas durante o trabalho de campo. Nas aldeias estudadas, havia 310 indígenas com idade $>20$ anos $(87,3 \%$ da população Suruí dessa faixa etária nas 11 aldeias). Mulheres gestantes $(n=23)$ e indivíduos com deficiência física que impossibilitasse a utilização do esfigmomanômetro $(\mathrm{n}=1)$ não foram avaliados. Dentre os 286 indivíduos elegíveis para o estudo, 35 não se encontravam nas aldeias por ocasião da pesquisa de campo. Foram avaliadas 251 pessoas (87,8\% dos indivíduos elegíveis).

A população apresentou proporções semelhantes de homens e mulheres (50,6\% de mulheres). A média de idade da população foi de 36,9 anos (DP: 15,5), sendo de 36,8 (DP: 15,4) anos para homens e de 37,1 (DP: 15,6) anos para as mulheres. A estrutura etária corresponde a de uma população jovem, com $64,1 \%$ (masculino: 64,5\%; feminino: 63,8\%) dos indivíduos com idade inferior a 40 anos. Não se verificou diferença estatisticamente significativa na distribuição por idade entre os sexos $\left(\chi^{2}=0,758\right)$ (Tabela 1$)$.

\section{Variáveis antropométricas}

\section{Peso e estatura}

A média de peso foi superior no sexo masculino (masculino: 65,9kg, DP: 10,5; feminino: $57,9 \mathrm{~kg}$, DP: 11,1), assim como a média de estatura (masculino: 160,0 cm, DP: 4,96; feminino: 147,2 cm, DP: 4,48).

\section{Demais indicadores antropométricos (IMC, PC, RCQ)}

As médias do IMC foram semelhantes entre os sexos (masculino: 22,6 kg/m² f feminino: 22,6 
Tabela 1. Frequências de indígenas Suruí com idade maior ou igual a 20 anos por faixa etária, estado nutricional e índice socioeconômico, segundo sexo, Rondônia, 2005.

\begin{tabular}{|c|c|c|c|c|c|c|c|}
\hline \multirow[b]{2}{*}{ Indicadores } & \multicolumn{2}{|c|}{ Masculino } & \multicolumn{2}{|c|}{ Feminino } & \multicolumn{2}{|c|}{ Total } & \multirow[b]{2}{*}{ Valor do $\mathrm{p}^{\star}$} \\
\hline & n & $\%$ & $\mathbf{n}$ & $\%$ & $\mathbf{n}$ & $\%$ & \\
\hline Faixa etária & & & & & & & 0,758 \\
\hline $20-29$ & 54 & 43,5 & 51 & 40,2 & 105 & 41,8 & \\
\hline $30-39$ & 26 & 21,0 & 30 & 23,6 & 56 & 22,3 & \\
\hline $40-49$ & 28 & 22,6 & 25 & 19,7 & 53 & 21,1 & \\
\hline 50 e mais & 16 & 12,9 & 21 & 16,5 & 37 & 14,7 & \\
\hline Total & 124 & 100,0 & 127 & 100,0 & 251 & 100,0 & \\
\hline IMC & & & & & & & 0,018 \\
\hline Baixo peso & 2 & 1,6 & - & 0 & 2 & 0,8 & \\
\hline Peso adequado & 53 & 42,7 & 55 & 43,3 & 108 & 43,0 & \\
\hline Sobrepeso & 57 & 46,0 & 44 & 34,6 & 101 & 40,2 & \\
\hline Obesidade & 12 & 9,7 & 28 & 22,0 & 40 & 15,9 & \\
\hline Total & 124 & 100,0 & 127 & 100,0 & 251 & 100,0 & \\
\hline RCQ & & & & & & & 0,000 \\
\hline Adequada & 57 & 46,0 & 19 & 15,0 & 76 & 30,3 & \\
\hline Elevada & 67 & 54,0 & 108 & 85,0 & 175 & 69,7 & \\
\hline Total & 124 & 100,0 & 127 & 100,0 & 251 & 100,0 & \\
\hline PC & & & & & & & 0,000 \\
\hline Adequado & 90 & 72,6 & 38 & 29,9 & 128 & 51,0 & \\
\hline Elevado & 34 & 27,4 & 89 & 70,1 & 123 & 49,0 & \\
\hline Total & 124 & 100,0 & 127 & 100,0 & 251 & 100,0 & \\
\hline SSE & & & & & & & 0,317 \\
\hline Inferior & 34 & 30,1 & 47 & 39,5 & 81 & 34,9 & \\
\hline Intermediário & 47 & 41,6 & 44 & 37,0 & 91 & 39,2 & \\
\hline Superior & 32 & 28,3 & 28 & 23,5 & 60 & 25,9 & \\
\hline Total & 113 & 100,0 & 119 & 100,0 & 232 & 100,0 & \\
\hline
\end{tabular}

Notas: $\left({ }^{*}\right)$ teste do qui-quadrado; Abreviações: IMC - Índice de Massa Corporal, RCQ - razão cintura-quadril; PC - perímetro da cintura; SSE - status socioeconômico.

$\left.\mathrm{kg} / \mathrm{m}^{2}\right)$. As médias de praticamente todos os demais indicadores antropométricos analisados foram superiores no sexo masculino ( $\mathrm{PC}$ - masculino: $88,8 \mathrm{~cm}$, DP: 9,5; feminino: $87,5 \mathrm{~cm}$, DP: 11,3; RCQ - masculino:0,95, DP:0,06; feminino: 0,92, DP: 0,07), exceto a da prega cutânea triciptal (PCT), única a apresentar diferença estatisticamente significativa entre os sexos (masculino:14,3cm, DP: 5,7; feminino: $27,0 \mathrm{~cm}$, DP: 8,3$)$.

Mais da metade dos adultos Suruí apresentou excesso de peso, sendo essa proporção semelhante entre os sexos $(p=0,867)$. As prevalências de obesidade ( $p=0,007)$, perímetro da cintura $(P C)(p<0,000)$ e de razão cintura/quadril (RCQ) elevados $(p<0,000)$ foram significativamente maiores entre as mulheres. As frequências de indivíduos por categorias dos indicadores antropométricos segundo sexo são apresentadas na Tabela 1.

\section{Indicador socioeconômico}

Cerca de $40 \%$ da população foi classificada no nível socioeconômico intermediário. A diferença entre os sexos quanto à distribuição da frequência de SSE não foi estatisticamente significativa, mas observa-se maior frequência de mulheres no nível socioeconômico inferior (Tabela 1).

\section{Pressão Arterial}

A PAS variou de $90 \mathrm{mmHg}$ a $163 \mathrm{mmHg}$, com média de 111,7 mmHg (DP: 11,08). A PAD variou de $46 \mathrm{mmHg}$ a $93 \mathrm{mmHg}$, com média de 69,8 mmHg (DP: 8,24). Ambas as médias (PAS e PAD) foram superiores no sexo masculino, embora sem significância estatística.

As médias de PAS e PAD e respectivos intervalos de confiança por faixa etária, indicadores nutricionais e SSE segundo sexo são apresentadas na Tabela 2. Verifica-se incremento da média de PAS no sexo masculino conforme aumenta a 
Tabela 2. Médias e desvios-padrão globais e por sexo de pressão arterial sistólica (PAS) e pressão arterial diastólica (PAD) segundo estratos de idade decenais, variáveis antropométricas e nível socioeconômico para indígenas Suruí com idade > 20 anos, Rondônia, 2005.

\begin{tabular}{|c|c|c|c|c|c|c|c|}
\hline \multirow[b]{3}{*}{ Indicadores } & \multicolumn{7}{|c|}{ PAS } \\
\hline & \multicolumn{3}{|c|}{ Masculino } & \multicolumn{3}{|c|}{ Feminino } & \multirow[b]{2}{*}{ Valor do $\mathrm{p}^{\star}$} \\
\hline & $\mathbf{n}$ & Média & IC $95 \%$ & $\mathbf{n}$ & Média & IC $95 \%$ & \\
\hline \multicolumn{8}{|l|}{ Faixa Etária } \\
\hline $20-29$ & 54 & 111,0 & $109,1-112,9$ & 51 & 109,8 & $107,3-112,2$ & 0,410 \\
\hline $30-39$ & 26 & 113,8 & $109,4-118,3$ & 30 & 107,0 & $103,7-110,3$ & 0,012 \\
\hline $40-49$ & 28 & 112,3 & $106,8-117,9$ & 25 & 113,8 & $107,1-120,6$ & 0,720 \\
\hline 50 e mais & 16 & 112,8 & $108,1-117,4$ & 21 & 117,5 & $111,1-124,0$ & 0,246 \\
\hline Total & 124 & 112,1 & $110,4-113,9$ & 127 & 111,2 & $109,1-113,3$ & 0,490 \\
\hline \multicolumn{8}{|l|}{ IMC } \\
\hline Peso adequado & 53 & 112,6 & $109,5-115,6$ & 55 & 112,04 & $108,1-116,0$ & 0,834 \\
\hline Sobrepeso & 57 & 110,9 & $108,6-113,3$ & 44 & 109,36 & $106,7-112,0$ & 0,375 \\
\hline Obesidade & 12 & 116,6 & $110,1-123,1$ & 28 & 112,39 & $108,3-116,5$ & 0,252 \\
\hline \multicolumn{8}{|l|}{ RCQ } \\
\hline Adequada & 57 & 110,4 & $108,1-112,6$ & 19 & 111,2 & $106,6-115,9$ & 0,718 \\
\hline Elevada & 67 & 113,6 & $111,0-116,3$ & 108 & 111,2 & $108,8-113,6$ & 0,187 \\
\hline \multicolumn{8}{|l|}{$\mathrm{PC}$} \\
\hline Adequado & 90 & 111,9 & $109,9-113,9$ & 38 & 108,4 & $105,3-111,6$ & 0.061 \\
\hline Elevado & 34 & 112,7 & $108,7-116,7$ & 89 & 112,4 & $109,7-115,1$ & 0,894 \\
\hline \multicolumn{8}{|l|}{ SSE } \\
\hline Inferior & 34 & 113,1 & $109,3-117,0$ & 47 & 111,2 & $107,7-114,7$ & 0,465 \\
\hline Intermediário & 47 & 112,4 & $110,1-114,8$ & 44 & 112,5 & $108,3-116,8$ & 0,952 \\
\hline \multirow[t]{3}{*}{ Superior } & 32 & 112,2 & $108,2-116,3$ & 28 & 109,6 & $105,6-113,7$ & 0,355 \\
\hline & \multicolumn{7}{|c|}{ PAD } \\
\hline & \multicolumn{3}{|c|}{ Masculino } & \multicolumn{3}{|c|}{ Feminino } & \\
\hline Indicadores & $\mathbf{n}$ & Média & IC 95\% & $\mathbf{n}$ & Média & IC 95\% & Valor do $\mathrm{p}^{*}$ \\
\hline \multicolumn{8}{|l|}{ Faixa Etária } \\
\hline $20-29$ & 54 & 70,9 & $69,1-72,8$ & 51 & 66,7 & $64,1-69,3$ & 0,007 \\
\hline $30-39$ & 26 & 72,0 & $68,7-75,3$ & 30 & 70,2 & $67,6-72,9$ & 0,393 \\
\hline $40-49$ & 28 & 71,5 & $68,2-74,9$ & 25 & 70,6 & $67,0-74,3$ & 0,711 \\
\hline 50 e mais & 16 & 67,3 & $62,0-72,5$ & 21 & 69,5 & $66,3-72,7$ & 0,421 \\
\hline Total & 124 & 70,8 & $69,4-72,2$ & 127 & 68,8 & $67,3-70,3$ & 0,049 \\
\hline \multicolumn{8}{|l|}{ IMC } \\
\hline Peso adequado & 53 & 69,0 & $67,0-71,1$ & 55 & 67,2 & $64,7-69,7$ & 0,266 \\
\hline Sobrepeso & 57 & 71,5 & $69,3-73,6$ & 44 & 69,9 & $67,9-71,8$ & 0,291 \\
\hline Obesidade & 12 & 76,3 & $71,6-81,1$ & 28 & 70,1 & $66,5-73,6$ & 0,044 \\
\hline \multicolumn{8}{|l|}{ RCQ } \\
\hline Adequada & 57 & 69,5 & $67,8-71,3$ & 19 & 66,6 & $63,1-70,2$ & 0,114 \\
\hline Elevada & 67 & 71,9 & $69,8-74,1$ & 108 & 69,1 & $67,5-70,8$ & 0,041 \\
\hline \multicolumn{8}{|l|}{$\mathrm{PC}$} \\
\hline Adequado & 90 & 69,7 & $68,1-71,2$ & 38 & 65,5 & $62,6-68,3$ & 0,006 \\
\hline Elevado & 34 & 73,8 & $70,8-76,9$ & 89 & 70,2 & $68,5-71,8$ & 0,028 \\
\hline \multicolumn{8}{|l|}{ SSE } \\
\hline Inferior & 34 & 70,5 & $67,5-75,6$ & 47 & 67,2 & $64,5-69,9$ & 0,107 \\
\hline Intermediário & 47 & 71,1 & $69,2-73,0$ & 44 & 70,5 & $68,3-72,7$ & 0,661 \\
\hline Superior & 32 & 71,8 & $68,7-74,9$ & 28 & 68,9 & $65,3-72,4$ & 0,213 \\
\hline
\end{tabular}

Notas: $\left.{ }^{*}\right)$ Teste t student; Abreviaçoes: IMC - Índice de Massa Corporal, RCQ - razão cintura-quadril; PC - perímetro da cintura; SSE - status socioeconômico; $\mathrm{DP}=$ Desvio Padrão. 
idade até os 40 anos, enquanto no sexo feminino, as médias de PAS mais elevadas foram verificadas nos estratos de idade acima de 40 anos. As médias masculinas de PAS são superiores às femininas nos estratos mais jovens, fato que se inverte a partir dos 40 anos, quando as médias femininas superam as masculinas. A faixa etária de 30 a 39 anos foi a única a apresentar diferença estatisticamente significativa entre as médias de PAS segundo sexos.

Em relação à $\mathrm{PAD}$, verifica-se médias masculinas decrescentes a partir dos 30 anos, ao passo que entre as mulheres nota-se incremento das médias até os 49 anos. A média de PAD feminina só supera a masculina no estrato de idade de 50 anos e mais. A faixa etária de 20 a 29 anos foi a única a apresentar diferença estatisticamente significativa entre as médias de PAD segundo sexos.

As maiores médias de PAS e PAD foram encontradas entre os indivíduos obesos, em ambos os sexos. Não houve diferenças estatisticamente significativas entre as médias por sexo de PAS e PAD entre os estratos de IMC. As médias de PAS e PAD foram mais elevadas no sexo masculino, em todos os estratos de IMC. O estrato de obesidade foi o único a apresentar diferença estatisticamente significativa entre as médias de PAD segundo sexos.

Em relação à RCQ e ao $\mathrm{PC}$, todas as médias de PAS e PAD foram maiores nos estratos considerados elevados, com exceção da PAS feminina para a RCQ. Verificou-se média de PAD signifi- cativamente mais elevada no sexo masculino no estrato de RCQ elevada. Verificou-se ainda, diferença significativa entre os sexos em ambas as categorias de PC e média de PAD significativamente mais elevada no estrato de PC elevado, entre as mulheres.

Em relação ao SSE, não se verificou diferença estatisticamente significativa entre as médias de PAS e PAD entre os diferentes estratos socioeconômicos, em ambos os sexos.

A prevalência global de hipertensão arterial foi de $2,8 \%$, sendo o valor verificado entre as mulheres $(3,1 \%)$ superior aos homens $(2,4 \%)$. Nenhum homem foi classificado no estágio de maior gravidade (Estágio 2), enquanto uma mulher foi classificada neste estágio.

A prevalência de HAS foi maior nas faixas etárias mais elevadas, globalmente e no sexo masculino. Nas mulheres, só se verificou hipertensão arterial acima dos 40 anos de idade. A prevalência de HAS foi maior em indivíduos com concentração abdominal de gordura (PC e RCQ elevados) e nos estratos de menor nível socioeconômico (Tabela 3).

As matrizes de correlação de Pearson segundo sexos são apresentadas na Tabela 4. Nos homens, a PAS correlacionou-se significativamente com a PAD e com a RCQ. Ressalta-se a fraca correlação da PAS com a idade, bem como as correlações inversas, ainda que não significativas com perímetro do quadril (PQ), PCT e área de gordura do braço (AGB). A PAD correlacionou-

Tabela 3. Prevalências de nível tensional indicativo de HAS segundo estratos de indicadores nutricionais e socioeconômicos, indígenas Suruí com idade > 20 anos, Rondônia, 2005.

\begin{tabular}{|c|c|c|c|c|c|c|}
\hline \multirow[b]{2}{*}{ Indicadores/estratos } & \multicolumn{2}{|c|}{ Homens } & \multicolumn{2}{|c|}{ Mulheres } & \multicolumn{2}{|c|}{ Total } \\
\hline & $\mathbf{n}$ & $\%$ & n & $\%$ & $\mathbf{n}$ & $\%$ \\
\hline \multicolumn{7}{|l|}{ Idade } \\
\hline$<30$ anos & 54 & 0,0 & 51 & 0,0 & 105 & 0,0 \\
\hline 30 a 39 anos & 26 & 3,8 & 30 & 0,0 & 56 & 1,8 \\
\hline$>40$ anos & 44 & 4,5 & 46 & 8,7 & 90 & 6,7 \\
\hline \multicolumn{7}{|l|}{ Estrato Nutricional } \\
\hline $\mathrm{IMC}<25$ & 74 & 3,6 & 67 & 7,3 & 141 & 5,5 \\
\hline IMC $>25$ & 50 & 1,4 & 60 & 0,0 & 110 & 0,7 \\
\hline \multicolumn{7}{|l|}{ Concentração abdominal de gordura } \\
\hline PC adequado & 90 & 2,2 & 38 & 0,0 & 128 & 1,6 \\
\hline PC elevado & 34 & 2,9 & 89 & 4,5 & 123 & 4,1 \\
\hline RCQ adequado & 57 & 0,0 & 19 & 0,0 & 76 & 0,0 \\
\hline RCQ elevado & 67 & 4,5 & 108 & 3,7 & 175 & 4,0 \\
\hline \multicolumn{7}{|l|}{ Nível sócio-econômico } \\
\hline I/II (inferior) & 92 & 2,2 & 99 & 4,0 & 191 & 3,1 \\
\hline III (superior) & 32 & 3,1 & 28 & 0,0 & 60 & 1,7 \\
\hline
\end{tabular}

Notas: Abreviações: IMC - Índice de Massa Corporal, RCQ - razão cintura-quadril; PC - perímetro da cintura; HAS: Hipertensão Arterial Sistêmica; JNC ${ }^{26}$ 
Tabela 4. Matriz de correlação de Pearson para os adultos Suruí de ambos os sexos, 2005.

\begin{tabular}{|c|c|c|c|c|c|c|c|c|c|c|c|c|c|}
\hline & Idade & PAS & PAD & Estatura & Peso & IMC & PC & RCQ & PQ & PB & PCT & AMB & AGB \\
\hline Idade & & $0,272^{* *}$ & 0,133 & $-0,041$ & $-0,036$ & $-0,032$ & $0,269^{* *}$ & $0,597^{* *}$ & $-0,099$ & $-0,017$ & $-0,136$ & $0,200^{*}$ & $-0,099$ \\
\hline PAS & 0,057 & & $0,486^{* *}$ & $-0,048$ & 0,045 & 0,061 & $0,209^{*}$ & $0,269^{* *}$ & 0,024 & $-0,011$ & 0,04 & $-0,072$ & 0,04 \\
\hline PAD & $-0,171$ & $0,517^{* *}$ & & $-0,156$ & $0,200^{*}$ & $0,261^{* *}$ & $0,289^{* *}$ & $0,211^{*}$ & $0,220^{*}$ & $0,254^{* *}$ & $0,276^{* *}$ & 0,027 & $0,285^{* *}$ \\
\hline Estatura & $-0,116$ & $-0,149$ & 0,024 & & $0,338^{* *}$ & 0,011 & 0,08 & $-0,143$ & $0,254^{* *}$ & 0,167 & $-0,003$ & $0,302^{* *}$ & 0,059 \\
\hline Peso & $-0,266^{* *}$ & 0,011 & $0,281^{* *}$ & $0,423^{* *}$ & & $0,942^{* *}$ & $0,825^{* *}$ & $0,318^{* *}$ & $0,956^{* *}$ & $0,911^{* *}$ & $0,780^{* *}$ & $0,427^{* *}$ & $0,863^{* *}$ \\
\hline IMC & $-0,246^{* *}$ & 0,077 & $0,303^{* *}$ & 0,038 & $0,919^{* *}$ & & $0,846^{* *}$ & $0,384^{* *}$ & $0,925^{* *}$ & $0,908^{* *}$ & $0,830^{* *}$ & $0,344^{* *}$ & $0,894^{* *}$ \\
\hline $\mathrm{PC}$ & 0,018 & 0,097 & $0,299^{* *}$ & 0,114 & $0,877^{* *}$ & $0,918^{* *}$ & & $0,742^{* *}$ & $0,801^{* *}$ & $0,779^{* *}$ & $0,697^{* *}$ & $0,323^{* *}$ & $0,744^{* *}$ \\
\hline RCQ & & $0,183^{*}$ & & $-0,177^{*}$ & & & & & $0,220^{*}$ & $0,348^{* *}$ & $0,304^{* *}$ & 0,167 & $0,316^{* *}$ \\
\hline PQ & $-0,290^{* *}$ & $-0,025$ & $0,249^{* *}$ & $0,329^{* *}$ & $0,939^{* *}$ & $0,896^{* *}$ & $0,845^{* *}$ & $0,339^{* *}$ & & $0,863^{* *}$ & $0,762^{* *}$ & $0,365^{* *}$ & $0,830^{* *}$ \\
\hline $\mathrm{PB}$ & $-0,223^{*}$ & 0,06 & $0,288^{* *}$ & 0,137 & $0,860^{* *}$ & $0,889^{* *}$ & $0,826^{* *}$ & $0,528^{* *}$ & $0,808^{* *}$ & & $0,836^{* *}$ & $0,496^{* *}$ & $0,922^{* *}$ \\
\hline PCT & $-0,299^{* *}$ & $-0,038$ & $0,268^{* *}$ & 0,043 & $0,732^{* *}$ & $0,795^{* *}$ & $0,712^{* *}$ & $0,377^{* *}$ & $0,769^{* *}$ & $0,730^{* *}$ & & $-0,06$ & $0,979^{* *}$ \\
\hline AMB & $-0,004$ & 0,126 & 0,136 & 0,167 & $0,490^{* *}$ & $0,461^{* \star}$ & $0,460^{* *}$ & $0,374^{* *}$ & $0,376^{* *}$ & $0,689^{* *}$ & 0,008 & & 0,131 \\
\hline AGB & $-0,280^{* *}$ & $-0,012$ & $0,287^{* *}$ & 0,07 & $0,796^{* *}$ & $0,852^{* *}$ & $0,769^{* *}$ & $0,424^{* *}$ & $0,810^{* *}$ & $0,819^{* *}$ & $0,987^{* *}$ & 0,152 & \\
\hline
\end{tabular}

Nota: $\square$ Masculino Feminino. ${ }^{*}$ ) p-valor $\left.<0,05 ; 2 .{ }^{* *}\right)$ p-valor $<0,01$; Abreviações: PAS - Pressão arterial sistólica, PAD - pressão arterial diastólica, IMC - índice de massa corporal, PC - perímetro da cintura, RCQ - razão cintura/quadril, $\mathrm{PQ}$ - perímetro do quadril, $\mathrm{PB}$ perímetro braquial, PCT - prega cutânea triciptal, AMB - área muscular do braço, AGB - área de gordura do braço.

Tabela 5. Comparação dos valores de média da pressão arterial sistólica e diastólica e prevalência de hipertensão entre os anos de 1988 e 2005, para ambos os sexos.

\begin{tabular}{|c|c|c|c|c|c|c|}
\hline \multirow{3}{*}{ Indicadores } & \multicolumn{4}{|c|}{ Ano do estudo } & \multirow{3}{*}{$\begin{array}{l}\text { Diferenças } \\
\text { entre os anos }\end{array}$} & \multirow{3}{*}{ Valor do $\mathrm{p}^{*}$} \\
\hline & \multicolumn{2}{|r|}{$1988^{*}$} & \multicolumn{2}{|r|}{2005} & & \\
\hline & $\mathbf{n}$ & Média (DP) & $\mathbf{n}$ & Média (DP) & & \\
\hline \multicolumn{7}{|l|}{ Média de PAS } \\
\hline Média Global & 114 & - & 251 & $111,7(11,1)$ & - & \\
\hline Média masculina & 60 & $110,7(11,5)$ & 124 & $112,1(10,0)$ & 1,4 & 0,390 \\
\hline Média feminina & 54 & $103,3(8,3)$ & 127 & $111,2(12,0)$ & 7,9 & 0,000 \\
\hline \multicolumn{7}{|l|}{ Média de PAD } \\
\hline Média Global & 114 & - & 251 & $69,8(8,2)$ & - & \\
\hline Média masculina & 60 & $69,6(7,0)$ & 124 & $70,8(8,0)$ & 1,2 & 0,320 \\
\hline \multirow[t]{3}{*}{ Média feminina } & 54 & $66,6(8,2)$ & 127 & $68,8(8,4)$ & 2,2 & 0,106 \\
\hline & \multicolumn{2}{|c|}{ Prevalência $1988^{\star}$} & \multicolumn{2}{|c|}{ Prevalência 2005} & & \\
\hline & $\mathbf{n}$ & $\%$ & $\mathbf{n}$ & $\%$ & & \\
\hline \multicolumn{7}{|l|}{ Prevalência de HAS } \\
\hline Global & 0 & 0,0 & 7 & 2,8 & $2,8 \%$ & - \\
\hline Masculino & 0 & 0,0 & 3 & 2,4 & $2,4 \%$ & - \\
\hline Feminino & 0 & 0,0 & 4 & 3,1 & $3,1 \%$ & - \\
\hline
\end{tabular}


presente estudo, que são comparadas às reportadas no estudo realizado no ano de 1988 com a mesma população ${ }^{15}$. Observa-se que houve uma elevação da média de PAS e PAD entre os anos comparados em ambos os sexos, com maior diferença na PAS para o sexo feminino (7,9 mmHg), sendo a única a apresentar significância estatística $(p=0,000)$. Em 1988, não foram encontrados casos de hipertensão arterial entre os Suruí, ao passo que por ocasião da coleta de dados do presente estudo, a prevalência global de hipertensão arterial foi de 2,8\%.

\section{Discussão}

A hipertensão arterial encontra-se na lista das mais frequentes morbidades em todo o mundo, estando relacionada ao rápido processo de transição epidemiológica e nutricional em curso em uma escala global, incluindo os povos indígenas.

Há poucos estudos sobre a epidemiologia da hipertensão arterial em povos indígenas no Brasil. Até o início dos anos 90, estudos realizados em populações que ainda apresentavam um padrão de alimentação caracterizado por baixa ingestão de gorduras e ausência de sódio, em sua maioria situadas na Amazônia, reportaram médias de pressão arterial sistólica e diastólica abaixo das verificadas na população brasileira não indígena, assim como ausência de casos de hipertensão arterial ${ }^{6,8,23,24}$.

Em 1988, os Suruí foram avaliados pela primeira vez em relação à pressão arterial, confirmando o que vinha sendo relatado para povos amazônicos $^{6}$. No intervalo de dezessete anos, verificou-se incremento da PAS e da PAD em ambos os sexos, sendo este mais pronunciado no sexo feminino, com significância estatística para a elevação de PAS nas mulheres. Nas duas ocasiões (1988 e 2005) as médias de PAS e PAD foram superiores no sexo masculino, mas as diferenças das médias entre os sexos diminuiu entre os anos comparados, passando respectivamente de 7,4 $\mathrm{mmHg}$ e 3,0 mmHg, em 1988, para 0,9 mmHg e $2,0 \mathrm{mmHg}$, em 2005. Ainda que tenha sido verificada elevação dos níveis tensionais médios, a prevalência de hipertensão arterial Suruí foi de 2,8\%, inferior à verificada na população não indígena brasileira, na qual a prevalência de HAS varia de $22 \%$ a $44 \%$ em áreas urbanas ${ }^{25-27}$. Apesar disso, esta é a primeira vez que são reportados casos de hipertensão arterial em indígenas Suruí.

Ainda que a prevalência de hipertensão entre estes tenha sido baixa em relação aos grupos com- parados, ressalta-se que nenhum dos indivíduos investigados fazia uso de medicação anti-hipertensiva por ocasião da coleta de dados, pois não vinham sendo acompanhados pelo serviço local de saúde.

A comparação direta das médias de PAS e PAD Suruí entre os dois inquéritos realizados na etnia deve ser feita com cautela, já que não foi possível fazer a padronização por idade. No intervalo entre as duas investigações, a população Suruí pode ter sofrido mudanças na estrutura etária, possivelmente retratando uma estrutura de população menos jovem na coleta mais recente, sendo esperadas, portanto, médias mais elevadas de PAS e PAD. A mesma cautela deve ser tomada com relação à comparação direta da prevalência de hipertensão arterial Suruí com as de outras populações indígenas e não indígenas, pois ainda que possa ter havido um envelhecimento populacional, os Suruí permanecem com estrutura etária jovem $(64,1 \%$ com idade inferior a 40 anos), sendo esperadas menores prevalências desse agravo. Esta situação é corroborada pela prevalência mais elevada de hipertensão arterial no grupo etário com idade maior a 40 anos $(6,7 \%)$. Comparações mais acuradas necessitariam de padronização por idade.

As médias de PAS e PAD verificadas no presente estudo entre os Suruí superam as encontradas em outras etnias indígenas no Bra$s 1^{8,23,28,29}$. As médias de PAS e PAD de indígenas Suruí do sexo masculino, reportadas em 1988, já superavam as médias ponderadas correspondentes para 11 grupos indígenas sul-americanos ${ }^{30}$, ao passo que as médias femininas Suruí eram inferiores. Na presente análise, as médias femininas se aproximam das masculinas e ambas ultrapassam os valores reportados para os 11 grupos, em $1988^{30}$. A superioridade atual dos níveis tensionais médios Suruí pode ser justificada, em parte, por diferenças temporais na coleta dos dados. É possível que as populações sul-americanas estudadas à época apresentem, atualmente, níveis tensionais mais elevados e/ou superiores aos Suruí, haja vista a amplitude e a rapidez do processo de transição epidemiológica e nutricional em curso que afeta profundamente os povos indígenas em território nacional.

Entre os Suruí, a prevalência de hipertensão arterial foi maior no sexo feminino, ao contrário do que frequentemente é descrito em populações não indígenas ${ }^{27,31,32}$. Estes resultados coincidem com aqueles reportados para outros povos indígenas no Brasil. Entre os Xavantes de Mato Grasso, a prevalência de HAS foi de $7,7 \%$ e de $5,3 \%$ 
em mulheres e homens, respectivamente ${ }^{8}$. Nos Guarani no Estado do Rio de Janeiro, essas prevalências foram de 7,4\% e 2,6\% ${ }^{29}$ e, entre os Tupiniquim no Espírito Santo, de 19,3\% e 17,6\%7 . Nesses estudos, as diferenças de níveis tensionais e prevalências de hipertensão arterial entre os sexos têm sido atribuídas a diferenças na composição corporal, tal como concentração abdominal de gordura, assim como a distintos padrões de consumo alimentar e de atividade física.

Em diversas populações nativas da Amazônia ${ }^{6}$ verificou-se ausência de associação positiva entre idade e níveis tensionais. No estudo realizado com os Suruí no final da década de 1980, a PAS apresentava-se inversamente associada à idade em ambos os sexos ${ }^{15}$. No presente estudo, detectou-se correlação positiva e estatisticamente significativa entre idade e PAS no sexo feminino, ao passo que a PAD mostrou-se inversamente associada à idade no sexo masculino, ainda que não estatisticamente significativa. A comparação dos resultados obtidos nos dois estudos realizados entre os Suruí em um intervalo de aproximadamente dezessete anos evidencia que houve uma inversão no sentido da associação entre pressão arterial e idade, particularmente no sexo feminino, indicando a existência de um processo de transição em saúde entre os Suruí, vivenciado de forma distinta entre os sexos.

À semelhança do observado por Bloch et al. ${ }^{29} \mathrm{e}$ Cardoso et al. ${ }^{28}$, as correlações positivas das variáveis antropométricas (RCQ, PC, IMC, peso) obtidas entre os Suruí, sobretudo a concentração abdominal de gordura, com a PAS e PAD, indicam que o aumento nos níveis pressóricos, pelo menos em parte, está associado ao acúmulo de gordura corporal, especialmente a de deposição central.

O perfil epidemiológico da população Suruí é caracterizado por apresentar altas prevalências de doenças infecciosas e parasitárias ${ }^{11,16}$ e desnu- trição infantil ${ }^{10}$, elevadas taxas de fecundidade ${ }^{33}$, ao mesmo tempo em que se observa o aumento das prevalências de sobrepeso/obesidade em adultos $^{13,14}$ e de hipertensão arterial.

Ao longo das últimas três décadas, os Suruí passaram por profundas mudanças em suas práticas de produção e consumo de alimentos, vindo a consumir alimentos ricos em gordura, sal e açúcar. As roças e outras atividades de subsistência como a caça, pesca e coleta de produtos florestais, são praticamente inexistentes para parcela expressiva da população atual, o que ocasiona diminuição da atividade física ${ }^{13,15,34}$. Conforme descrito anteriormente, esses fatores, agindo em conjunto, teriam importante papel na redefinição dos padrões de adoecimento e o perfil epidemiológico do grupo, abrindo espaço para o surgimento de doenças crônicas.

\section{Considerações Finais}

Os resultados apontam para a necessidade de elaboração de estratégias para prevenir e controlar os fatores de risco para hipertensão arterial e outras doenças e agravos não transmissíveis, particularmente no grupo feminino. Para isso, seria necessário o planejamento e a implementação de ações que visem discutir e sensibilizar os Suruí acerca das relações entre padrões de consumo alimentar, particularmente o consumo de sal, açúcar, alimentos industrializados, gorduras, e de atividade física e DANT. Além disso, devem ser realizados estudos para identificar outros fatores de risco associados às DANT, a fim de subsidiar estratégias mais amplas de prevenção e promoção à saúde, pautadas em ações sustentáveis de subsistência, tendo em vista os recursos disponíveis no território, a história de contato e os modos atuais de interação com o mercado regional. 


\section{Colaboradores}

FG Tavares participou da coleta de dados, da concepção do artigo da análise e interpretação dos dados, da redação, revisão crítica e aprovação da versão final do artigo. CEA Coimbra Júnior participou na coleta de dados, delineamento do estudo, na interpretação dos dados, na redação e revisão crítica do artigo e na aprovação de sua versão final. AM Cardoso participou da concepção do artigo, na interpretação dos dados, na redação e revisão crítica do artigo e na aprovação de sua versão final.

\section{Agradecimentos}

Aos Suruí, pelo apoio durante a pesquisa de campo. Ao Cassius Schnel Palhano Silva pela realização dos exames pressóricos e ao Conselho nacional de Desenvolvimento Científico e Tecnológico $(\mathrm{CNPq})$, pelo apoio financeiro ao projeto.

\section{Referências}

1. Coimbra Júnior CEA, Santos RV. Emerging health needs and epidemiological research in indigenous peoples in Brazil. In: Salzano FM, Hurtado AM, editors. Lost Paradises and the Ethics of Research and Publication. Oxford: Oxford University Press; 2004.

2. Gimeno SGA, Rodrigues D, Canó EN, Lima EES, Schaper M, Pagliaro H, Lafer MM, Baruzzi RG. Cardiovascular risk factors among Brazilian Karib indigenous peoples: Upper Xingu, Central Brazil, 2000-3. J Epidemiol Community Health 2009; 63(4):299-304.

3. Cardoso AM, Mattos IE, Koifman RJ. Prevalência de diabetes mellitus e da síndrome de resistência insulínica nos índios Guaraní do Estado do Rio de Janeiro. In: Coimbra Júnior. CEA, Santos RV, Escobar AL, organizadores. Epidemiologia e Saúde dos Povos Indígenas no Brasil. Rio de Janeiro: Editora Fiocruz; 2003. p. 169-185.

4. Oliveira GF, Oliveira TRR, Rodrigues FF, Corrêa LF, Ikejiri AT, Casulari LA. Prevalência de diabetes melito e tolerância à glicose diminuída nos indígenas da Aldeia Jaguapiru, Brasil. Rev Panam Salud Publica 2011; 29(5):315-321.

5. Rocha AKS, Bós AJG, Huttner E, Machado DC. Prevalência da síndrome metabólica em indígenas com mais de 40 anos no Rio Grande do Sul, Brasil. Rev Panam Salud Publica 2011; 29(1):41-45.

6. Fleming-Moran M, Coimbra Júnior CEA. Blood pressure studies among Amazonian native populations: A review from an epidemiological perspective. Soc Sci Med 1990; 31(5):593-601.

7. Meyerfreund D, Gonçalves CP, Cunha RS, Pereira AC, Krieger JE, Mill JG. Age-dependent increase in blood pressure in two different Native American communities in Brazil. J Hypertens 2009; 27(9):17531760 .

8. Coimbra Júnior. CEA, Chor D, Santos RV, Salzano FM. Blood pressure levels in Xavante adults from the Pimentel Barbosa Indian Reserve, Mato Grosso, Brasil. Ethn Dis 2001; 11(2):232-240.

9. Orellana JDY, Basta PC, Santos RV, Coimbra Júnior CEA. Morbidade hospitalar em crianças indígenas Suruí menores de dez anos, Rondônia, Brasil: 2000 a 2004. Rev Bras Saúde Mater Infant 2007; 7(3):281287.

10. Orellana JDY, Coimbra Júnior CEA, Lourenço AEP, Santos RV. Estado nutricional e anemia em crianças Suruí, Amazônia, Brasil. Jornal de Pediatria 2006; 82(5):383-388.

11. Palhano-Silva CS, Araújo AJG, Lourenço AEP, Bastos OMP, Santos RV, Coimbra Júnior CEA. Intestinal parasitic infection in the Suruí Indians, Brazilian Amazon. Interciencia 2009; 34(4):259-264.

12. Basta PC, Coimbra Júnior CEA, Camacho LAB, Santos RV. Risk of tuberculous infection in an indigenous population from Amazonia, Brazil. Int $J$ Tuberc Lung Dis 2006; 10(12):1354-1359. 
13. Santos RV, Coimbra Júnior CEA. Socioeconomic differentiation and body morphology in the suru of Southwestern Amazonia. Curr Anthrop 1996; 37(5):851-856.

14. Lourenço AEP, Santos RV, Orellana JDY, Coimbra Júnior CEA. Nutrition transition in Amazonia: Obesity and socioeconomic change in the Suruí Indians from Brazil. Amer J Hum Biol 2008; 20(5):564-571.

15. Fleming-Moran M, Santos RV, Coimbra Júnior. CEA. Blood pressure levels of the Suruí and Zoró Indians of the Brazilian Amazon: group- and sex-specific effects resulting from body composition, health status, and age. Hum Biol 1991; 63(6):835-861.

16. Basta PC, Coimbra Júnior CEA, Escobar AL, Santos RV, Alves LCC, Fonseca LdS. Survey for tuberculosis in an indigenous population of Amazonia: the Suruí of Rondônia, Brazil. Trans R Soc Trop Med Hyg 2006; 100(6):579-585.

17. Fuchs FD, Gus M, Moreira WD, Moreira LB, Moraes RS, Rosito GA, Sorucco A, Atanázio P, Machado R. Blood pressure effects of antihypertensive drugs and changes in lifestyle in a Brazilian hypertensive cohort. J Hypertens 1997; 15(7):783-792.

18. Chobanian AV, Bakris GL, Black HR, Cushman WC, Green LA, Izzo JL, Jones DW, Materson BJ, Oparil S, Wright JT Jr, Roccella EJ. Seventh report of the Joint National Committee on Prevention, Detection, Evaluation, and Treatment of High Blood Pressure. Hypertension 2003; 42(6):1206-1252.

19. Lohman TG, Roche AF, Martorell R. Anthropometric Standardization Reference Manual. Champaign: Human Kinetics; 1988.

20. Frisancho AR. New norms of upper limb fat and muscle areas for assessment of nutritional status. Am J Clin Nutr 1981; 34(11):2540-2545.

21. World Health Organization (WHO). Physical Status: the Use and Interpretation of Anthropometric Indicators of Nutritional Status. Geneve: WHO; 1995

22. World Health Organization (WHO). Obesity: Preventing and Managing the Global Epidemic. Geneva: WHO; 2000.

23. Pavan L, Casiglia E, Braga LMC, Winnicki M, Puato M, Pauletto P, Pessina AC. Effects of a traditional lifestyle on the cardiovascular risk profile: the Amondava population of the Brazilian Amazon. Comparison with matched African, Italian and Polish populations. J Hypertens 1999; 17(6):749-756.

24. Lowenstein FW. Blood-pressure in relation to age and sex in the tropics and subtropics: a review of the literature and an investigation in two tribes of Brazil Indians. Lancet 1961; 277(7173):389-392.

25. Gus I, Harzheim E, Zaslavsky C, Medina C, Gus M. Prevalência, reconhecimento e controle da hipertensão arterial sistêmica no estado do Rio Grande do Sul. Arq Bras Cardiol 2004; 83(5):424-428.
26. Mion Júnior D, Gomes MAM, Nobre F, Amodeo C, Kohlmann Júnior O, Praxedes JN, Machado CA. IV Diretrizes Brasileiras de Hipertensão Arterial. Arq Bras Cardiol 2004; 82(Supl. 4):7-14.

27. Lessa Î. Epidemiologia da hipertensäo arterial sistêmica e da insuficiência cardíaca no Brasil. Revista Brasileira de Hipertensão 2001; 8(4):383-392.

28. Cardoso AM, Mattos IE, Koifman RJ. Prevalência de fatores de risco para doenças cardiovasculares na população Guaraní-Kaiowá do Estado do Rio de Janeiro. Cad Saude Publica 2001; 17(2):345-354.

29. Bloch KV, Coutinho ESF, Lôbo MEC, Oliveira JEP, Milech A. Pressão arterial, glicemia capilar e medidas antropométrica em uma população Yanomámi. Cad Saude Publica 1993; 9(4):428-438.

30. Salzano FM, Callegari-Jacques SM. South American Indians: a Case Study in Evolution. Oxford: Clarendon Press; 1988.

31. Burt VL, Whelton P, Roccella EJ, Brown C, Cutler JA, Higgins M, Horan MJ, Labarthe D. Prevalence of hypertension in the US adult population. Results from the third National Health and Nutrition Examination Survey, 1988-1991. Hypertension 1995; 25(3):305-313.

32. Lolio CA, Pereira JCR, Lotufo PA, Souza JMP. Hipertensão arterial e possíveis fatores de risco. Rev Saude Publica 1993; 27(5):357-362.

33. Valencia MMA, Santos RV, Coimbra Júnior CEA, Oliveira MVGd, Escobar AL. Aspectos de la fecundidad de mujeres indígenas Suruí, Rondônia, Brasil: una aproximación. Rev Bras Saúde Mater Infant 2010; 10(3):349-358.

34. Santos RV, Coimbra Júnior CEA. Hardships of contact: Enamel hypoplasias in Tupi-Monde amerindians from the Brazilian Amazonia. Am J Phys Anthropol 1999; 109(1):111-127.

Artigo apresentado em 29/05/2012

Aprovado em 15/06/2012

Versão final apresentada em 06/07/2012 